\title{
Aplicación de la Metodología de Investigación de Casos de Estudio a Pequeñas y Medianas Empresas Pymes del País
}

\author{
Rodolfo A. Bergantino K. ${ }^{1}$ y María Fuensanta Donoso ${ }^{2}$ \\ 1 Profesor e Investigador, Facultad de Negocios, Universidad Católica Santa María la Antigua (USMA), \\ Panamá, República de Panamá. \\ 2 Profesora e Investigadora, Facultad de Negocios, Universidad Católica Santa María la Antigua (USMA), \\ Panamá, República de Panamá. \\ *Autor para correspondencia. Email: rbergantino@usma.ac.pa
}

Recibido: 07 de septiembre de 2017

Aceptado: 05 de octubre de 2017

\begin{abstract}
Resumen: El documento describe el desarrollo de casos de estudio como investigación aplicada producto de un marco teórico básico y la experiencia de los investigadores y la viabilidad de su uso como herramienta académica y de vinculación. En concreto, el escrito describe la experiencia de la investigación aplicada recientemente, que produjo dos estudios de casos de pyme panameñas. La investigación anterior incluyó además de la escritura de los casos, la propuesta de una guía básica para la redacción de casos de estudio, que se utilizará con fines académicos. Los casos fueron validados para el aprendizaje y la enseñanza por grupos de estudiantes y pares académicos. Además de compartir esta experiencia que produjo la guía básica, consideramos importante explorar el desarrollo de casos de estudio como una herramienta para la vinculación de la Universidad y la Comunidad. Por lo tanto, este trabajo, además de explorar las opiniones de los dos primeros grupos sobre el uso de casos para fines enseñanza-aprendizaje, se complementa con las opiniones de un grupo de empresarios de PYMES.
\end{abstract}

\section{Palabras claves. Casos de estudio, pymes,}

\begin{abstract}
The paper describes the process of developing case studies for academic purposes within panamanian University, acccording to previous training and practically experience of the researchers. Specifically, it describes the experience of the applied research that produced two cases studies of Panamanian SMEs. The previous research included besides the cases, the proposal of a basic guide for writing case studies to be used for academic purposes. The cases utillity for learning and teaching purposes were validated by groups of students, and academic peers. In addition, besides sharing this experience that produced the basic guide, we considered important to explore the development of these case studies as a tool for University and Community
\end{abstract}


Invest. pens. crit. (ISSN 1812-3864)

Vol. 5, No. 3, septiembre-diciembre 2017

pp. $5-18$

engagement. Therefore, this paper, in addition to exploring the opinions of the first two groups on the use of case studies for academic purposes, it is complemented by a focus group lead of SME's entrepreneurs, who explore the potential of fostering the development of this type of applied research..

\section{Keywords. Case studies, pymes,}

\section{Introducción}

En enero de 2016 propusimos la realización de un estudio sobre la "Aplicación de la Metodología de Investigación de Casos de Estudio a Pequeñas y Medianas Empresas PYMES del País" a la Dirección de Investigación de la USMA, deseando, entre otras cosas, fomentar el debate sobre el uso, desarrollo y aplicación de metodologías de investigación cualitativas, participativas y descriptivas como herramientas disponibles dentro del campo de las ciencias económicas. Este tipo de investigación se ha extendido a raíz de los cambios en el paradigma educativo surgido con el advenimiento de lo que algunos consideran el advenimiento de la $4^{\mathrm{a}}$ revolución industrial: la era del conocimiento basada en las plataformas digitales, la inteligencia artificial y el internet de las cosas (Schwab, 2016)

En consecuencia, este artículo trata sobre algunas de las experiencias y aprendizajes que vivimos como investigadores en nuestro ánimo de aplicar la metodología de casos de estudio para identificar y describir algunas situaciones del desarrollo competitivo en Pymes del entorno nacional y de su uso potencial como elemento de vinculación entre la universidad y las Pymes. Nos interesaba determinar la existencia o ausencia de algunos factores endógenos o exógenos que estuvieran contribuyendo al desarrollo de Pymes de algunos sectores de interés económico y de qué forma la universidad podría vincularse con ellas.

Algunos de los factores de desarrollo que analizamos dentro de la investigación incluían, los siguientes:

- Utilización de las TIC's en sus procesos administrativos

- La capacidad de expandirse hacia mercados internacionales.

- La capacidad de crear productos y/o servicios únicos o diferentes a lo tradicional.

- La capacidad de innovación técnica, administrativa, productiva y/o de procesos.

- La capacidad de generar valor agregado a clientes internos y externos por medio de la calidad del servicio y atención al cliente.

- La capacidad de generar y administrar cadenas de suministros de una forma eficiente.

- La capacidad de generar valor mediante la innovación social y la asociatividad empresarial.

Además, en el ámbito académico, teníamos el objetivo de validar los casos y la guía didáctica con profesores y estudiantes de pre y post grado. También se pensó en convocar a gerentes de empresas Pymes, que leyeran los casos y analizaran si servían para el desarrollo de competencias gerenciales específicas y genéricas dentro del ámbito empresarial. Sin embargo, se identificó la falta de un relacionamiento establecido con estos grupos para poder inclusive continuar con la escritura de casos a futuro, por lo que se pensó en el tema del focus group como una herramienta de 
Invest. pens. crit. (ISSN 1812-3864)

Vol. 5, No. 3, septiembre-diciembre 2017

pp. $5-18$

exploración, el punto de vista de los empresarios sobre la utilidad en general de la escritura de casos y la vinculación con la universidad para estos y otros objetivos.

\section{Desarrolllo del método de caso de estudios en la Administración:}

El método de casos de estudio, fue desarrollado en la Facultad de Derecho de la Universidad de Harvard se propagó dentro del mundo de las ciencias administrativas, primero en la Escuela de Negocios de la misma Universidad y luego en muchas otras.

"Posteriormente fue la Universidad de Chicago (Hamel et al., 1993) la que lideró el empleo de esta metodología de investigación. Sin embargo, este esfuerzo sirvió para generar muy poca teoría y debate académico (Rumelt et al., 1994), por lo que su eficacia como herramienta de investigación fue muy limitada y cuestionada y perdió aceptación dentro de la comunidad científica a favor de métodos cuantitativos de mayor objetividad, confiabilidad, validez y representatividad, más cercanos a las ciencias experimentales." (Villareal y Landeta, 2010).

Este modelo positivista de pensamiento e investigación recibió una creciente ola de críticas durante la segunda mitad del Siglo XX. Esto se debió al monismo metodológico que conlleva, al presuponer que todo investigador es objetivo y que puede por medios exclusivamente experimentales medir, describir y representar el contexto que lo rodea. Para los investigadores inclinados por este método de pensamiento la realidad observable, sólo se puede cuantificar, explicar y predecir por medio de la correlación entre diversas variables claves.

Para López y Salas (2009) los métodos de investigación utilizados por los investigadores positivistas...

“...a menudo se olvidan de aspectos importantes que se encuentran dentro del contexto de la investigación. En particular, señalan que las medidas cuantitativas imponen significados e interpretaciones externas a los datos, excluyendo de esta forma significados e interpretaciones que los miembros del grupo social estudiado atribuyen a la realidad observada".

Bernal (2010) sostiene la necesidad de incorporar en la investigación en ciencias sociales y en economía la complejidad y lo multidisciplinario. Basado en una extensa bibliografía, él afirma que el modelo tradicional de pensamiento científico, con prevalencia de lo objetivo sobre lo subjetivo, no alcanza para tratar de explicar la realidad multidimensional, caótica e inestable que se vive en el entorno de estas ciencias hoy día. Según Bernal...

“...para el paradigma de la complejidad, el conocimiento científico es una de las diversas formas de conocer el mundo, pero no la única. Y el concepto de saber es más adecuado que el concepto de conocimiento y más todavía que el de conocimiento científico." 
Invest. pens. crit. (ISSN 1812-3864)

Vol. 5, No. 3, septiembre-diciembre 2017

pp. $5-18$

Citando a Ruiz (1997), Bernal (2010) nos presenta algunas de estas características de la complejidad del mundo moderno, de entre las cuales podemos destacar las siguientes sin ningún orden predeterminado:

- La visión de la complejidad implica la apertura metodológica oponiéndose al mecanismo metodológico y al reduccionismo positivista.

- La complejidad integra al observador como parte de lo observado.

- Acepta lo constante pero también el cambio.

- Reconoce la comprensión del desorden, el error, la contradicción, y la incertidumbre.

- Rompe con el sentido unilineal de la evolución al incorporar el principio de la retroalimentación o la recursividad.

Como investigadores del conocimiento aplicado a la administración de negocios, sostenemos que al momento de estudiar una empresa con fines de orientarla en una dirección determinada debemos hacer un análisis holístico, yendo más allá del enfoque cuantitativo. Para ello debemos analizar las múltiples relaciones complejas que existen entre las experiencias y las actitudes de los diferentes sujetos que la conforman. Además, la naturaleza profunda y compleja del entorno PESTLE ${ }^{1}$ que rodea a la empresa, obliga a examinar su realidad política, económica, social, tecnológica, legal y ambiental, lo cual requiere una visión más amplia que la propone por si sólo el modelo filosófico positivista.

\section{El proceso de escritura de un caso}

Para Lane (2007), la tarea de escritura de un caso es un proceso de seis (6) etapas que inicia con la decisión del escritor de usar un caso y termina con el uso del caso en el aula de clases. Para ello, sugiere un proceso de escritura de seis etapas adoptado de Leenders \& Ersking, el cual va desde la decisión del educador de utilizar un caso en clase hasta la obtención de los permisos necesarios para la utilización del caso.

\section{Metodología}

Los autores preferimos utilizar un esquema de siete etapas integradas en tres (3) fases tal como se muestra en la figura $\mathrm{N}^{\circ} 1$. Este es el esquema que proponemos en la Guía Práctica para la Escritura y Utilización del Método de Casos en las Facultades de Negocios ${ }^{2}$

\footnotetext{
1 Para una revisión más detallada del entorno PESTLE revisar FME (2007) en www.free-managementebooks.com

2 Documento parte del informe final de la investigación titulada "Aplicación de la Metodología de Investigación de Casos de Estudio a Pequeñas y Medianas Empresas PYMES del País” realizada por los autores entre los años 2016 y 2017.
} 
Invest. pens. crit. (ISSN 1812-3864)

Vol. 5, No. 3, septiembre-diciembre 2017

pp. 5-18

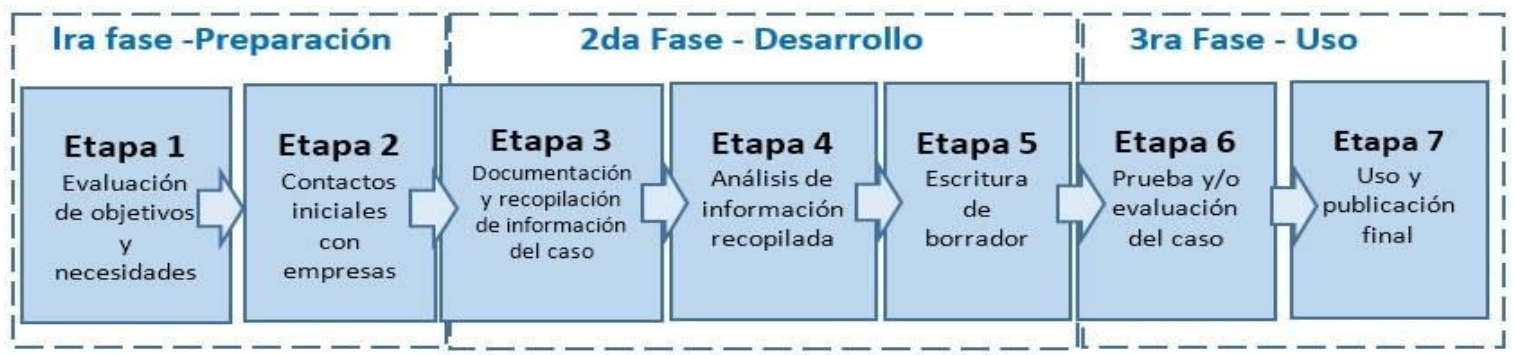

Figura No. 1. Etapas Básicas de Escritura de Casos.

Bergantino y Donoso 2017

La primera fase es de preparación, dentro de la cual se incorporan las dos etapas de la organización inicial para la escritura, esquivando obstáculos y evitando repeticiones. En esta primera fase preparatoria se realizan, además, los contactos iniciales con las empresas para determinar que en las empresas seleccionadas se pueden encontrar los factores de desarrollo que se necesitan para lograr el cumplimiento de los objetivos iniciales planteados en esta etapa.

En la segunda fase de desarrollo se tabula, resume, analiza, jerarquiza y organiza la información para iniciar el proceso de escritura de la siguiente etapa. Una vez escrito un borrador utilizable se inicia el proceso de prueba y uso del caso, lo que se hace en la $3^{\mathrm{a}}$ y última fase de acuerdo al diagrama. En esta tercera y última fase se prueba y valida el caso de estudio entre pares académicos, estudiantes y de ser posible con empresarios y/o gerentes del mismo tipo de empresas sobre las cuales estamos investigando. Finalmente el caso se publica y se usa con los fines académicos establecidos.

A continuación veremos cada una de las etapas de las fases mencionadas en detalle y de acuerdo al marco teórico utilizado, así como los aspectos concretos descriptivos de su desarrollo.

\section{Etapa N¹: Evaluación de los objetivos y necesidades de la investigación}

Villareal y Landeta (2010), proponen que en vista de que el método de casos es una metodología empírica se debe prestar particular atención a definir de antemano muy claramente los objetivos del estudio para luego no generar un conflicto de interpretación debido a la gran cantidad de información que se va a recopilar. Ellos sugieren que la aplicación del método de casos es útil para explorar y plantearse alternativas de explicación fenomenológica de situaciones sobre las cuales no se tiene un marco teórico muy bien delimitado.

El propósito de la investigación en este particular fue desarrollar conocimiento aplicado sobre pymes del país investigando situaciones de desarrollo empresarial, competitivo, innovador y asociativo, y su posible impacto en el desarrollo socioeconómico del país. Se utilizó una metodología para investigación cualitativa, exploratoria, descriptiva, por medio de la documentación de casos de estudio de Empresas pymes del país con características de asociatividad 
Invest. pens. crit. (ISSN 1812-3864)

Vol. 5, No. 3, septiembre-diciembre 2017

pp. $5-18$

e innovación empresarial. El Muestreo fue por aspectos lógicos, no probabilístico y a conveniencia de los investigadores y en relación directa con los objetivos de la investigación.

Así pues, para realizar esta investigación los autores decidimos realizar un estudio exploratorio, descriptivo, preliminar y explicativo sobre dos Pymes nacionales sobre cada una de las cuales se escribiría un caso de estudio. Escogimos una muestra lógica, no aleatoria, de empresas que tuvieran las características de internacionalización, innovación, generación de valor agregado eficiencia y asociatividad que ya hemos mencionado.

Para escoger las empresas revisamos las bases de datos de varios gremios de pymes del país y construimos fichas técnicas con información de las empresas que se mostraron anuentes a participar después de haberles presentado nuestros objetivos de investigación. También nos reunimos con algunos expertos nacionales e internacionales en temas de innovación y asociatividad empresarial, y les pedimos que nos refirieran con empresas Pymes modelos. De esta forma, construimos una base de datos con doce empresas que, por poseer algunas de las características deseadas, serían consideradas.

De forma paralela, los investigadores nos planteamos un protocolo formal para el estudio de cada empresa. Así determinamos la cantidad y el tipo de información que pretendíamos recoger de cada empresa y, además, la forma de intervención en cada una de ellas. Decidimos hacer un cuestionario con preguntas abiertas para facilitar el desarrollo del tema para los entrevistados. Además, acordamos hacer las entrevistas de forma conjunta para compartir el trabajo de observación, intervención y recogida de la información. Cabe mencionar que aunque en el diseño original de la investigación se determinó la necesidad y conveniencia de filmar las entrevistas, no fue posible realizar esto por razones presupuestarias.

Por otro lado, también elaboramos un memorándum de entendimiento que explicara a los empresarios el tipo de información que tendrían que compartir y la cantidad de tiempo aproximado que deberían dedicarle a las entrevistas. Con ellos abordamos los asuntos éticos más importantes tales como la confidencialidad de la información suministrada, la ausencia de una intención comercial de la investigación y el objetivo académico del mismo.

Sintéticamente podríamos resumir la información que se solicitaría a cada empresa de la siguiente forma:

a. Origen, desarrollo y evolución de la empresa.

b. Descripción de la situación actual de la empresa y de las principales expectativas.

c. Principales problemas que afronta en estos momentos.

d. Descripción de las principales áreas funcionales de la empresa, Mercadeo, Finanzas, Producción y Recursos Humanos.

e. Descripción del sector industrial en el cual está ubicada la empresa.

f. Relación con proveedores y clientes.

g. Relación con otras instituciones públicas y privadas. 
Invest. pens. crit. (ISSN 1812-3864)

Vol. 5, No. 3, septiembre-diciembre 2017

pp. 5-18

\section{Etapa N²: Establecimiento de los contactos iniciales con las empresas}

La etapa $\mathrm{N}^{\circ} 2$ del proceso consistió en el establecimiento de los contactos iniciales con las empresas. Cada investigador contaba ya en este momento con un universo de cinco empresas con las características que ya hemos mencionado anteriormente a partir de las cuales se haría un muestreo por conveniencia escogiendo a aquellas empresas que ofrecieran las mejores oportunidades de recopilar la información que se deseaba para escribir los dos casos de estudio.

De común acuerdo entre los investigadores, y después de revisar las fichas técnicas de cada una de las empresas, se escogieron las siguientes dos (2) empresas:

Empresa A: Empresa fundada en el año de 1997 y perteneciente al sector agroindustrial. Se dedicada principalmente al cultivo y procesamiento de palmito fresco y procesado. Posee más de 600 hectáreas en el área de Panamá Oeste. Esta empresa ha logrado exportar palmito a los selectivos mercados de Estados Unidos y de Europa gracias a la implementación de rigurosas medidas fitosanitarias de control de calidad por medio de un sistema de análisis de riesgos y puntos críticos de control.

Empresa B: Empresa del Sector Turismo fundada en el año 2005 y enfocada en la hospitalidad selectiva y campestre. Posee un hotel boutique/spa de 70 habitaciones en el área del Valle de Antón. Con su diseño arquitectónico toscano, cálido y acogedor, y basado en la naturaleza, es un referente del turismo en el área del Clúster Turístico de Coclé.

\section{Etapa N³: Recopilación y documentación de la información de campo}

Para realizar el proceso de recogida de la información de campo de tal forma que se pueda describir de una forma creíble la realidad observable dentro la empresa intervenida Villareal y Landeta (2010) proponen una serie de técnicas de recolección de evidencias como la recopilación de información documental tanto interna como externa de la empresa, y la realización de entrevistas abiertas con diversos informadores. En cuanto a la observación por parte del investigador ellos mencionan la posibilidad de incluir la observación participativa mediante la interacción dinámica del observador con el fenómeno bajo estudio.

Durante esta fase los autores intentamos mantener la validez constructiva de la investigación siguiendo un protocolo de intervención ajustado a cada una de las empresas de tal forma que pudiéramos mantener la cadena de evidencias de una forma ordenada y lógica. Además, tratamos en todo momento mantener la confiabilidad de la información obtenida mediante la realización de, al menos, tres técnicas de validación: en primer lugar la triangulación metodológica utilizando distintas metodologías de recopilación de la información, en segundo lugar la triangulación de investigadores, comparando constantemente nuestros apuntes y conclusiones, y finalmente la triangulación teórica, contrastando nuestros hallazgos con distintos enfoques teóricos administrativos. (Villareal y Landeta, 2010) 
Invest. pens. crit. (ISSN 1812-3864)

Vol. 5, No. 3, septiembre-diciembre 2017

pp. $5-18$

\section{Etapa $\mathrm{N}^{\circ}$ : Análisis de la información recopilada}

En cuanto a la investigación cualitativa Martínez Carazo (2006) afirma que no existe un procedimiento que se pueda considerar único para realizar el análisis inductivo de la información recogida durante el trabajo de campo. No obstante, sostiene que en una investigación de tipo cualitativa lo más importante es generar una comprensión cabal del problema bajo análisis mediante la aplicación de una lógica deductiva derivada de contrastar la información generada, con la bibliografía referente de nuestro marco teórico de investigación.

Para este trabajo investigativo y después de leer y releer la información recopilada en campo para familiarizarnos con el fenómeno administrativo y social que se estaba analizando, utilizamos varias herramientas de análisis, entre otras podemos destacar las siguientes:

- Resúmenes estructurados de la información.

- Diagramas de procesos.

- Matrices de relación y de ponderación o jerárquicas.

- Mapas conceptuales.

- Líneas de tiempo.

- Tablas y gráficas estadísticas.

- Análisis de contenidos.

Nuestro objetivo consistía en tratar de lograr una comprensión profunda de la situación analizada mediante discusión de nuestros análisis individuales con los otros miembros del equipo y con los entrevistados u observados. Una vez consideramos que habíamos identificado y conceptualizar correctamente el fenómeno estudiado, precedimos a la escritura de cada uno de los casos pertinentes.

\section{Etapa $N^{\circ}$ 5: Escritura del Borrador del Caso}

El proceso de escritura de un caso inicia con la firme convicción por parte del escritor de que el caso perfecto y completo no existe, y que el resultado inicial es un documento en borrador que necesita ser revisado y actualizado constantemente. Por lo tanto, es recomendable romper la inercia natural que nos impide iniciar la redacción del borrador inicial del caso, comenzando con la escritura del caso tan pronto tengamos una comprensión razonable de la fenomenología administrativa y social que ocurre dentro de la empresa bajo estudio. (Bergantino y Donoso 2017).

\section{Resultados. Discusión.}

Para escribir los casos referentes a esta investigación, decidimos utilizar un estilo narrativo directo libre, describiendo la trama del desde el punto de vista del tomador de decisiones, pero dejando que se escuchara en forma directa lo que pensaban sobre ciertos temas importantes otros protagonistas de la historia. Al final obtuvimos sendos casos de estudio contenidos en el informe 
Invest. pens. crit. (ISSN 1812-3864)

Vol. 5, No. 3, septiembre-diciembre 2017

pp. 5-18

final de la investigación los cuales serán publicados oportunamente. Estos son los dos (2) casos siguientes:

Caso de estudio: Los Mandarinos, Hotel Boutique/SPA ${ }^{3}$ Este caso describe el desarrollo de una empresa turística desde sus inicios, detalla los retos administrativos, técnicos y personales que tuvo que enfrentar para lograr desarrollarse y emprender el negocio de un hotel de oferta turística diferenciada que pudiera aprovechar la oferta escénica y de naturaleza; y propiciar la perspectiva de un desarrollo de marca región. Analiza, entre otros, aspectos administrativos, organizacionales, de asociatividad, desarrollo de marca región, financieros, sociales y culturales de la gestión de un negocio pyme en sus inicios en el sector turístico nacional

Caso de Estudio: Palmito S.A. ${ }^{4}$ El Caso Palmito Panamá es un caso clásico de toma de decisiones empresariales. El mismo aborda el desarrollo de una pequeña empresa agroindustrial desde sus inicios. Detalla los retos administrativos, técnicos y personales que la propietaria tuvo que enfrentar para lograr emprender y desarrollar el negocio de la exportación de palmito (palm heart). Analiza, entre otros, problemas administrativos, organizacionales, financieros, sociales y culturales de la gestión de un negocio agroindustrial y su relación con los mercados de exportación.

\section{Etapa Nº: Prueba y Evaluación de los casos}

Se precisa evaluar la validez del uso académico y el rigor metodológico de la escritura de los dos casos escritos mediante diversas herramientas de consulta. En nuestro caso se desarrollaron dos talleres de prueba del caso con estudiantes, un taller de evaluación de los casos con profesores y la viabilidad de uso y desarrollo de la herramienta con un focus group de empresarios.

Se parte de la premisa que se puede abordar el análisis de la opinión de un grupo, y asumir que sus comentarios pueden ser representativos de las instituciones o grupos generales que representan (estudiantes o profesores), si bien se respeta la individualidad de criterios que estos exponen (Bleger, 2007). Bajo este criterio mencionamos el análisis de los comentarios de los grupos de estudiantes.

Las técnicas de validación incluyeron una triangulación teórica, metodológica y de análisis de los investigadores a través de validación externa e interna de los casos de estudio mediante discusión con propietarios de las empresas; y con pares académicos, estudiantes de grado, postgrado y expertos consultores del área pyme.

\footnotetext{
${ }^{3}$ Documento aún no impreso producto final de la investigación titulada "Aplicación de la Metodología de Investigación de Casos de Estudio a Pequeñas y Medianas Empresas PYMES del País” realizada por los autores entre los años 2016 y 2017.

${ }^{4}$ Documento aún no impreso producto final de la investigación titulada "Aplicación de la Metodología de Investigación de Casos de Estudio a Pequeñas y Medianas Empresas PYMES del País" realizada por los autores entre los años 2016 y 2017.
} 
Invest. pens. crit. (ISSN 1812-3864)

Vol. 5, No. 3, septiembre-diciembre 2017

pp. $5-18$

\section{Validación con estudiantes}

El Caso de Estudio Hotel Los Mandarinos se discutió en un taller con 15 estudiantes de grado del curso Desarrollo de Emprendedores. Se les aplicó un formulario escrito con preguntas abiertas y cerradas. Además, se les hicieron preguntas abiertas orales y se motivó la participación de los estudiantes para que dieran su opinión sobre la forma y el fondo del caso. Las opiniones y los comentarios de los participantes así como sus recomendaciones se revisaron entre los investigadores y algunas de ellas fueron incorporadas al caso. Otra información se anotó como referencia para la realización de una futura actualización del caso.

El Caso de Estudio Palmito S.A. por su parte, fue analizado y discutido en clases con 15 estudiantes de maestría del curso Gerencia de Mercadeo. A los participantes se les aplicó un formulario escrito con preguntas abiertas. Se les preguntó su opinión sobre la forma y el fondo del caso y se les exhortó a comentar sobre la aplicabilidad del caso a cursos de grado y pregrado. Algunas de las recomendaciones después de haber sido consensuadas entre los investigadores fueron incorporadas al caso.

Los grupos de estudiantes valoran: el que sean casos reales, que sean de empresas de Panamá; pero de acuerdo a su nivel (licenciatura o posgrado) cuestionan longitud del material, y material adicional.

En general la respuestas de análisis del aprendizaje complejo por ellos mismo coincidió que el uso de casos de estudio es útil para el logro de aprendizajes complejo y más si existe conexión o identificación con la temática o el grupo analizado (Moran, 2006).

Los estudiantes de licenciatura declaran mayor interés en el uso de casos reales, y de empresas locales y que generan a su vez más elementos de discusión y análisis que a su vez llevan a aprendizaje prácticos. Por otro lado, valorarían el uso de video o fotos para ilustrar o explicar el caso. Un aspecto recomendado para la escritura de casos es que no se exponga toda la información y que se permita al estudiante inferir o investigar. Sin embargo, algunos estudiantes pidieron el que se diera información concreta o una orientación clara sobre lo que se plantea para investigar, pues la lectura comprensiva es un reto para ellos. Podemos tal vez determinar con estos comentarios que es recomendable el combinar la escritura redacción típica con elementos visuales como fotos, figuras o videos para unos estudiantes cada vez más familiarizados con herramientas multimedia. 
Invest. pens. crit. (ISSN 1812-3864)

Vol. 5, No. 3, septiembre-diciembre 2017

pp. $5-18$

\section{Estudiantes de Pre grado -Licenciatura}

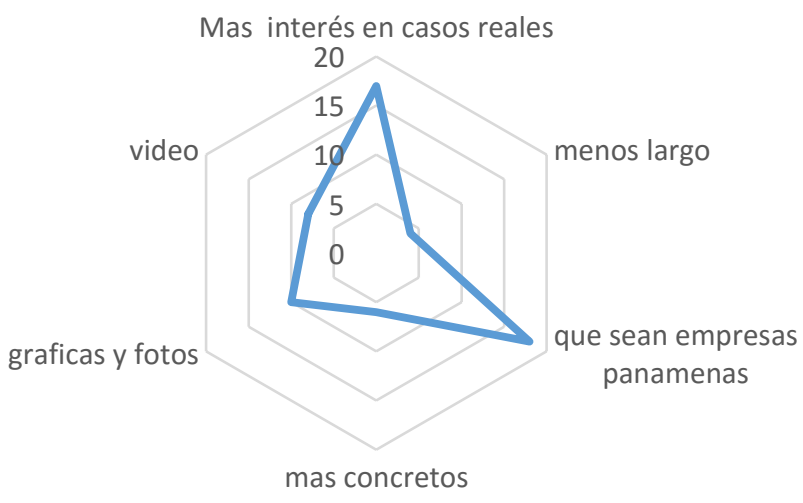

Gráfica 1 - Bergantino y Donoso 2017

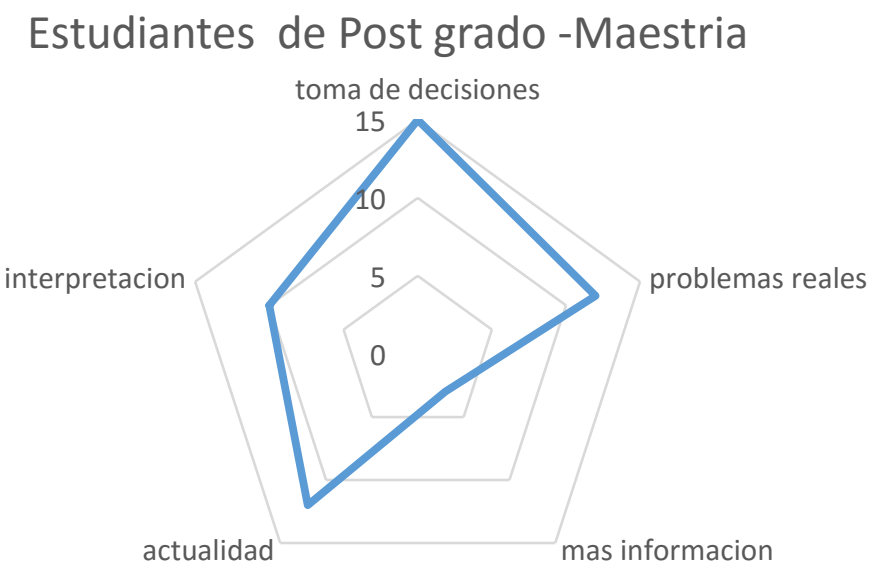

Gráfica 2 - Bergantino y Donoso 2017

\section{Validación con pares académicos.}

Se realizó un taller con 12 pares académicos de amplia experiencia en la utilización del método de casos y se discutieron a profundidad el rigor académico y la validez práctica de los casos de estudio escritos, tanto para cursos de licenciatura como de maestría.

Los grupos de profesore ven usabilidad en temas complementarios, proponen formatos predeterminados, seguimiento con un programa formal, etc. 
Invest. pens. crit. (ISSN 1812-3864)

Vol. 5, No. 3, septiembre-diciembre 2017

pp. $5-18$

Para el aprendizaje complejo y ejemplos prácticos. (Bartman, 2006).

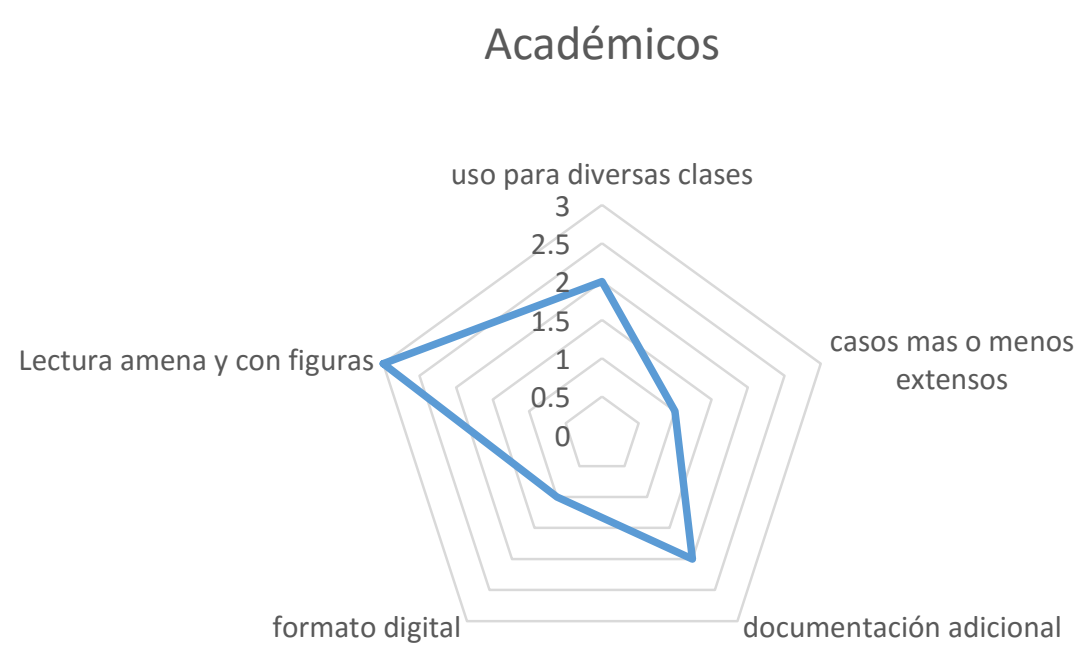

Gráfica 3 - Bergantino y Donoso 2017

Los académicos reportaron el uso conveniente de la guía para facilitar la aplicación de estos casos en particularar con fines académicos; y en general para el desarrollo de nuevos casos a futuro o su actualización.

\section{Validación con empresarios:}

Se realizó un cuarto taller en el formato de metodología de focus group con empresarios representantes y conocedores del ambiente PYME. Con ellos se discutió la validez externa de los casos en el sentido de su correspondencia entre los aspectos teóricos prácticos tratados en cada uno de ellos como en su pertinencia y relevancia con este sector de la economía nacional.

Los grupos de empresarios; reiteran que los casos de estudio deben contribuir al diagnóstico y desarrollo de las áreas operativas de la empresa y la planeación estratégica desde la cual la perspectiva de estudio de la toma de decisiones de los casos de estudio es interesante.

Por otro lado, mencionaron los empresarios, el desarrollo de acasos de estudio debe darse dentro del marco de fortalecimiento de programas más amplios y con objetivos complementarios (lutz, 2008). 
Invest. pens. crit. (ISSN 1812-3864)

Vol. 5, No. 3, septiembre-diciembre 2017

pp. $5-18$

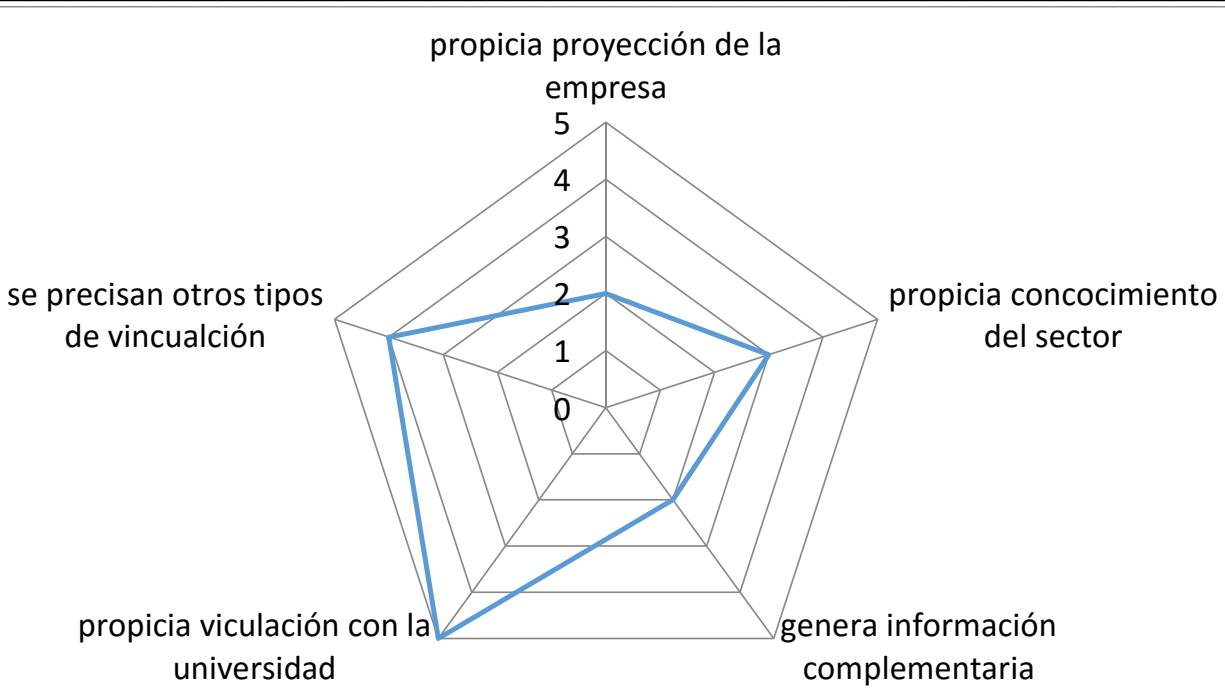

Gráfica 4 - Bergantino y Donoso 2017

\section{Conclusiones:}

Se pueden plantear algunas recomendaciones en base a la aplicación de los casos para fines académicos. El orden de desarrollo es plenamente utilizado por desarrolladores de casos en diversas universidades y las fases propuestas son una guía que puede ser adaptadas para diferentes profesores o investigadores interesados en el uso de casos para fines académicos o el desarrollo de nuevos casos.

Para la aplicación en clase de los casos de estudio, se utilizaran herramientas de análisis como mapas mentales, matrices de ponderación o la elaboración de gráficas o tablas, para analizar o explicar un concepto; además de la redacción de ensayos o trabajos escritos. Por otro lado, verificar que las gráficas o información que se puede incluir en el caso es claramente entendido por los estudiantes.

Es además valioso el referir o propiciar la búsqueda de libros de consulta y uso del recurso de la Internet para complementar la información ofrecida. Las TIC's como mencionan varios autores son herramientas que nos permiten acceder a gran cantidad ilimitada de información que pueden servir para complementar, adecuar y actualizar la información del caso, por lo que se considera importante incluir referencias de sitios de Internet.

Analizando la participación con el grupo de empresarios surgen recomendaciones de organización interna de la universidad para facilitar la escritura de casos y otros elementos de vinculación que pueden ser motivo de otra investigación en la búsqueda de modelos y mejores prácticas. En general, creemos necesario pues para profundizar el desarrollo de casos de estudio como herramientas académicas explorar también el marco de desarrollo de la vinculación comunidaduniversidad en especial con las pymes. 
Invest. pens. crit. (ISSN 1812-3864)

Vol. 5, No. 3, septiembre-diciembre 2017

pp. $5-18$

Reiteramos el valor del desarrollo y aplicación de casos de estudios para el logro de enseñanza aprendizaje, en particular de variable locales. Esto último es además es una herramientas de motivación y de contribución al papel de la universidad como ente científico social.

Se precisa continuar y profundizar en el uso de esta herramienta en otras facultades y universidades locales tal vez compartiendo experiencias y casos internacionales.

\section{Bibliografía}

Baartman, Bastiaens, Kirshner, \& Van der Vleuter, 2006 citado en: Proyecto Innova- Cesal, 2012. Estrategias para la Evaluación de Pensamiento Complejo y Competencias. Sistematización de experiencias y buenas prácticas de docentes universitarios. México. 2012.

Bleger, José. Psicología de la conducta. (2007). . Argentina: Paidós. p. 304.

FME, T. (14 de junio de 2017). wmw.free-management-ebooks.com. Obtenido de www.freemanagement-ebooks.com

Lane, J. L. (2007). www.schreyerinstitute.psu.edu. (P. State, Editor, \& S. I. Excellence, Productor) Obtenido de www.schreyerinstitute.psu.edu

López Herera, F., \& Salas-Haram, H. (2009). INVESTIGACIÓN CUALITATIVA EN ADMINISTRACIÓN. Cinta Moebio, 35, 128-145.

Villarreal Larrinaga, O., \& Landeta Rodríguez, J. (2010). EL ESTUDIO DE CASOS COMO METODOLOGÍA DE INVESTIGACIÓN CIENTÍFICA EN DIRECCIÓN Y ECONOMÍA DE LA EMPRESA. UNA APLICACIÓN A LA INTERNACIONALIZACIÓN. Investigaciones Europeas de Dirección y Economía de la Empresa, 16(3), 31-52.

Israel, B., Eng, E., Schulz, A.J., Parker, E.A. (Eds.). (2005). Methods in Community-Based Participatory Research for Health. San Francisco, CA: Jossey-Bass Publishers.

Lutz, J., \& Neis, B. (Eds.). (2008). Making and Moving Knowledge: Interdisciplinary and Community-Based Research in a World on the Edge. McGill-Queens University Press.

Moran Edgar. 1994. Introducción al pensamiento complejo España. Gedisa p. 176.

Schawb, Klaus. La Cuarta Revolución Industrial. Editorial Debate. España. 2016. 